CASE REPORT

\title{
Frontal Lobe Tuberculoma: A Clinical and Imaging Challenge
}

\author{
Tinsae Alemayehu ${ }^{1 *}$, Wondwossen Ergete ${ }^{2}$, Workeabeba Abebe ${ }^{1}$
}

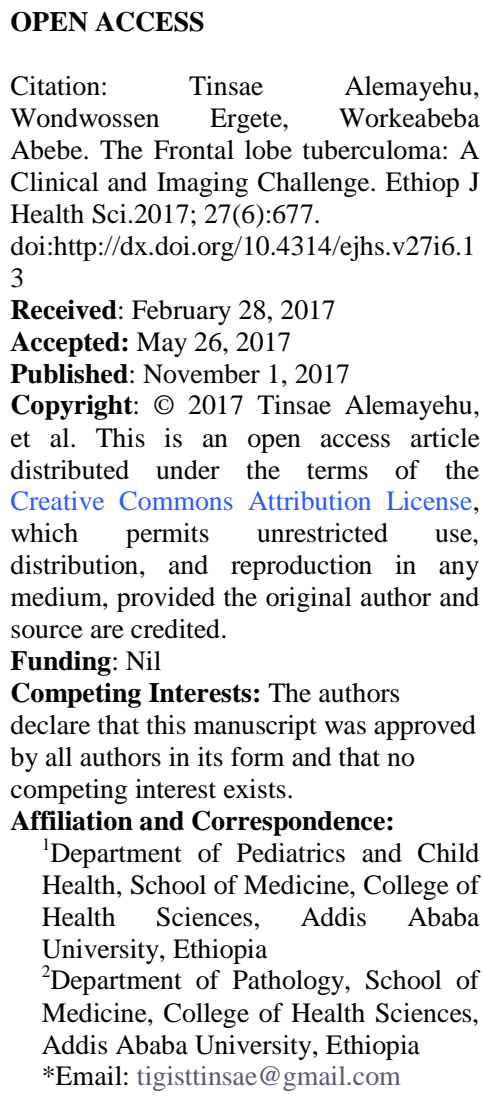

\begin{abstract}
BACKGROUND: Pediatric nervous system tuberculomas are usually infra-tentorial and multiple. A frontal lobe location is rare. CASE DETAILS: We report a 10 year-old boy who presented with a chronic headache and episodes of loss of consciousness. He had no signs of primary pulmonary tuberculosis and a diagnosis of frontal tuberculoma was made upon a post-operative biopsy. He improved following treatment with anti-tubercular drugs. CONCLUSION: Tuberculosis should be considered in children with a chronic headache or focal neurologic deficit and a supratentorial intracranial mass in endemic countries like Ethiopia.

KEYWORDS: Tuberculoma, Tuberculosis, Frontal lobe, Ethiopia

\section{INTRODUCTION}

Tuberculosis can involve various parts of the nervous system including the meninges, brain, spinal cord, cranial and peripheral nerves, ears and eyes. Hematogenous spread of Mycobacterium tuberculosis is the primary cause of tuberculosis of the brain (1). One group of manifestations of tuberculosis of the nervous system is tuberculomas. These are masses made of small tubercles with epitheloid cells at their core and surrounded by lymphocytes. They could be single or multiple. Tuberculomas are usually infra-tentorial and multiple in children. They are often found at the base of the brain (2). They consist of 5-8\% of operated intracranial masses in developing countries (3).

Tuberculomas can present in many ways with headache, seizures, localized neurologic deficits, behavioral problems and visual loss being some of the presenting complaints (4). They are usually diagnosed in the absence of systemic disease. Findings on imaging are non-specific (enhancing cystic lesions) and can mimic malignancies, brain abscesses, toxoplasmosis and hydatid disease (5).Tuberculomas occasionally present, or expand, during successful treatment of tuberculosis and be diagnosed as paradoxical reactions (6). We report a case of tuberculoma at an unusual site (frontal lobe) in a 10 year-old boy.

DOI: http://dx.doi.org/10.4314/ejhs.v27i6.13 


\section{CASE REPORT}

A 10 year-old boy presented to our hospital, with a global headache for 2 years with repeated episodes of loss of consciousness lasting for various durations. Six months before his presentation, he started having a left sided body weakness while his right side being unaffected. He also developed difficulties of vision and a progressive increase in head size. He had no other complaints. On physical examination, vital signs were found unaffected. His head circumference was $61 \mathrm{~cm}$ (greater than +2 standard deviation for his age). He had decreased visual acuity bilaterally with left sided hemiparesis. He was evaluated at a private clinic as possibly a brain malignancy and referred to Tikur Anbessa Specialized Hospital. Lab studies revealed a normal complete blood count and an ESR of $120 \mathrm{~mm} / \mathrm{hr}$. His chest $\mathrm{x}$-ray was normal (Figure 1).

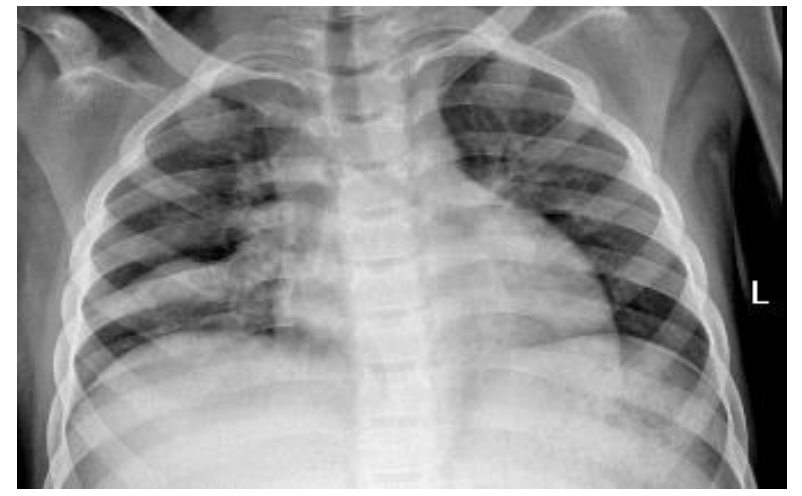

Figure 1: Chest X-ray of the child

A brain CT scan revealed a right fronto-parietal heterogeneously enhancing mass with calcifications and a significant mass effect (the differential diagnoses entertained were astrocytoma and a germ cell tumor) (Figure 2). The MRI of the child confirmed a large right frontal lobe intra-axial mass lesion with skull vault invasion likely a primitive neuro-ectodermal tumor (PNET).

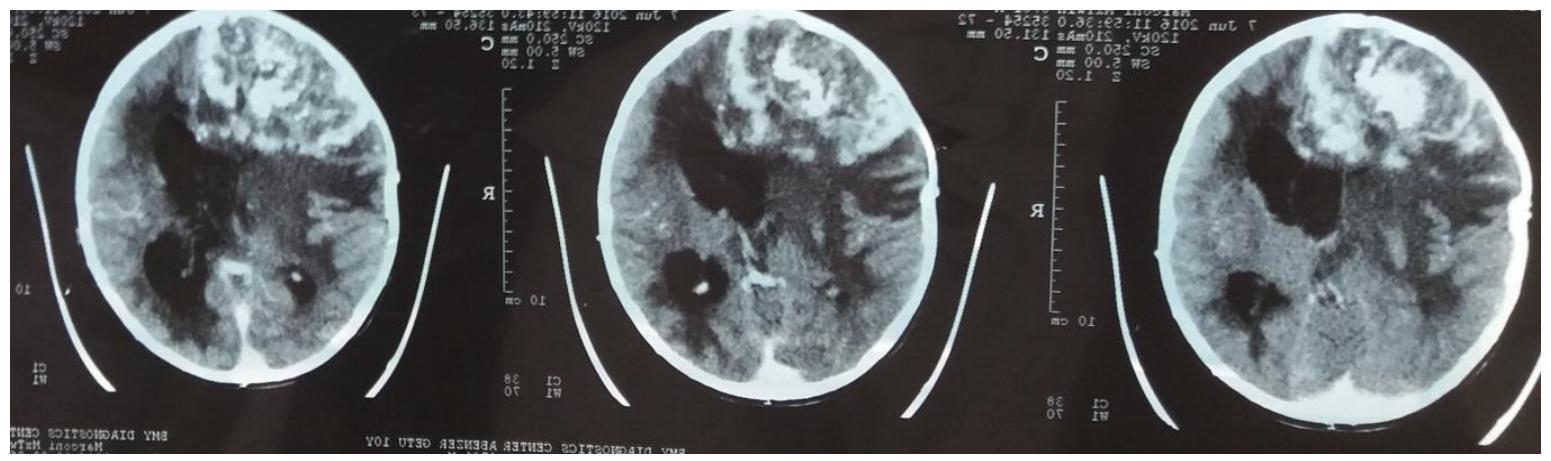

Figure 2: A right fronto-parietal heterogeneously enhancing mass with calcifications \& a mass effect

The boy underwent craniotomy, and a huge lobulated mass $(9.5 \times 8 \times 4 \mathrm{~cm})$ was excised and sent for a biopsy examination (Figure 3). Calcifications over the dura were noted. The mass was lobulated with a greyish tan, consisting of friable, yellowish tissue.

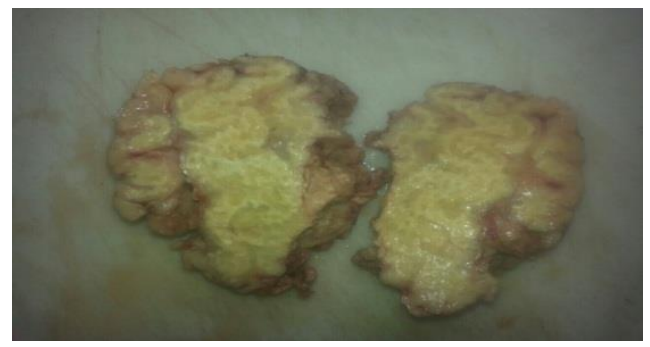

Figure 3: The excised mass 
The biopsy showed extensive areas of caseous necrosis surrounded by a variable amount of karrhectic debris and peripheral rim of fibrous tissue with infiltrates longemans type giant cells, epitheloid histiocytes, foamy macrophages, lymphocytes and some plasma cells. It confirmed the diagnosis of a right frontal tuberculoma (Figures 4 and 5).

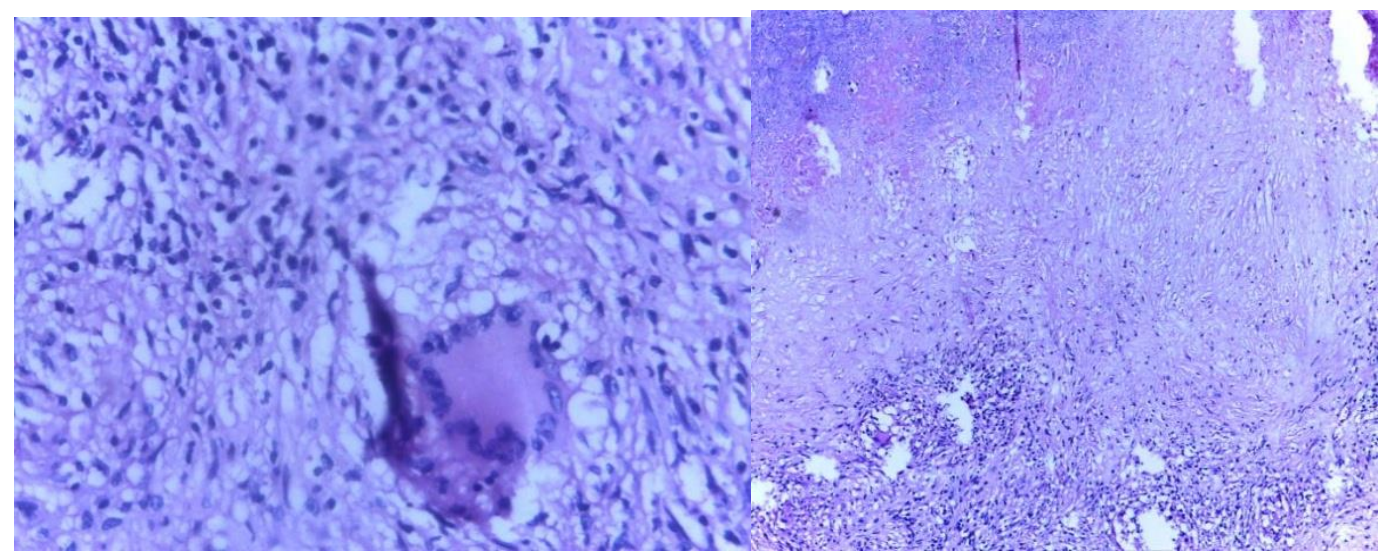

Figures 4 and 5: Microscopic features of excised mass

Our patient was initiated on anti-TB treatment (two months of isoniazid, rifampicin, pyrazinamide and ethambutol along with Prednisolone followed by a ten month continuation phase (isoniazid, rifampicin). He has completed his treatment and has shown a significant improvement of presenting symptoms, barring the poor visual acuity. He can only differentiate between light and darkness.

\section{DISCUSSION}

The neuroimaging of our patient showed a large right frontal mass with calcifications and a midline shift, an uncommon site for the illness. The chest x-ray was normal. As is often the case, the findings mimicked brain tumors such as an astrocytoma or a germ cell tumor. A biopsy confirmed a tuberculoma. Similar clinical courses, whereby a biopsy settled clinical and imaging dilemmas were reported among children in Iran, Saudi Arabia and Cote d'ivoire $(1,7,9)$. The frontal lobe localization of this tuberculoma is rare. In children, tuberculomas are usually diagnosed in the infra-tentorial region. They can present with chronic symptoms $(2,7)$. Clinical and imaging features are important to diagnose tuberculomas. However, CT findings of tuberculomas vary since they are evolving granulomas. As symptoms evolve, a normal CT, an enhancing hypo- or isodense lesion or a ring-like appearance may be seen. Differential diagnoses depend on the stage of lesion and can include cysticercosis, brain abscess, gliomas, etc (8). The variety of alternative diagnoses before histo-pathologic confirmation forces clinicians in the developing world to depend on epidemiologic patterns. Our patient's diagnosis was settled upon biopsy of the removed mass. The delay in diagnosis has contributed to his visual loss. Management of tuberculosis of the nervous system should be with a 4 drug regimen (Etambutol, Isoniazid, Rifampicin and Pyrazinamide) for 2 months followed by a 2 drug regimen (Isoniazid and Rifampicin) for 10 months. This regimen is accompanied by corticosteroids (10).

In conclusion, tuberculosis should be considered as one of the differential diagnoses for children presenting with a chronic headache or a focal neurologic deficit, even when the identified mass is Supra-tentorial. This should be the norm in countries endemic for Tuberculosis like Ethiopia.

\section{REFERENCES}

1. Saleh M, Saeedi A, Pooran A - Brain tuberculomas, a case report. Jundishapur $J$ Microbiol 2014; 7(7): 1- 3.

DOI: http://dx.doi.org/10.4314/ejhs.v27i6.13 
2. Pereira N, Shah I, Biyani N, Shah F - Frontal lobe tuberculoma. Oxford Medical Case Reports, 2016;1:12-14

3. Assefa G, Omar F, Biluts H, Abebe M. Review of the clinical, computerized tomography scan and/or magnetic resonance imaging findings of intra-cranial tuberculoma in an Ethiopian teaching hospital. Ethiop Med $J, 2016 ; 54: 3$.

4. Islam K, Rahman M, Akhter S, Alam S, Asaduzzaman K, Islam M - Tuberculoma of the brain: 3 case reports. JCMCTA 2010; 21 (2): 41-43.

5. Monteiro R, Carneiro J, Costa C, Duarte R Cerebral tuberculomas - a clinical challenge. Respiratory Medicine Case Reports, 2013;9:34-37.

6. Shah I, Borse $\mathrm{S}$ - Paradoxical tuberculomas after completion of anti-tuberculous treatment. Tropical medicine and health, 2012;40(1):1517.

7. Algahthani $\mathrm{H}$, Aldarmahi A, Algahthani A, Al-Rabia M, Samkari A - Tumor-like presentation of central nervous system tuberculosis: A retrospective study in Kingdom of Saudi Arabia; Journal of Taibah University Medical sciences, 2014);9:143-150.

8. Lwakatare F, Gabone J. Imaging features of brain tuberculoma in Tanzania: a case report and literature review. African health sciences 2003; 3(3):131-135.

9. N'da HA, Haidara A, Drogba L, Derou LK, Yao SK, Zeze V. A rare third ventricle solitary tuberculoma. Pan African med J, 2013; 16:5.

10. World Health Organization (WHO). Guidance for national tuberculosis programmes on the management of tuberculosis in children. World Health Organization.2014. 